\title{
The relationship between numerosity perception and mathematics ability in adults: The moderating role of dots number
}

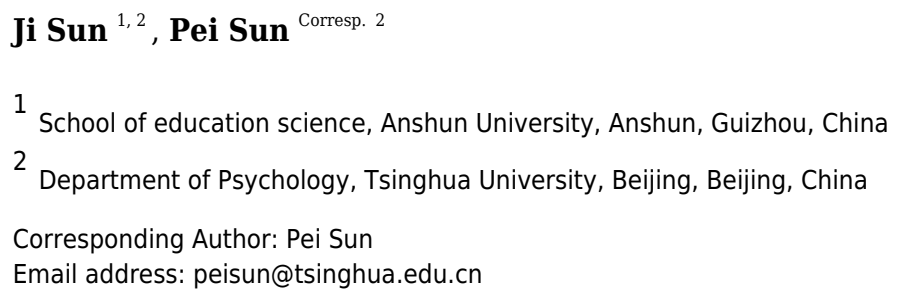

Background. It has been proposed that numerosity perception is the cognitive underpinning of mathematics ability. However, the existence of the association between numerosity perception and mathematics ability is still under debate, especially in adults. The present study examined the relationship between numerosity perception and mathematics ability and the moderating role of dots number (i.e., the numerosity of items in dot set) in adults. Methods. Sixty-four adult participants from Anshun University completed behavioral measures that tested numerosity perception of small numbers and large numbers, mathematics ability, inhibition ability, visual-spatial memory, and setswitching ability. Results. We found that numerosity perception of small numbers correlated significantly with mathematical ability after controlling the influence of inhibition ability, visual-spatial memory, and set-switching ability, but numerosity perception of large numbers was not related to mathematics ability in adults. Conclusions. These findings suggest that the dots number moderates the relationship between numerosity perception and mathematics ability in adults and may contribute to explaining the contradictory findings in the previous literature about the link between numerosity perception and mathematics ability. 
1 The relationship between numerosity perception and 2 mathematics ability in adults: The moderating role of 3 dots number

4

5

6

7

8

\author{
Ji Sun ${ }^{1,2}$, Pei $\operatorname{Sun}^{2}$ \\ ${ }^{1}$ School of education science, Anshun University, Anshun, Guizhou, China \\ ${ }^{2}$ Department of Psychology, Tsinghua University, Beijing, Beijing, China
}

Corresponding Author:

Pei Sun ${ }^{2}$

Weiqing Building, Tsinghua University, Haidian, Beijing, 100084, China

Email address: peisun@tsinghua.edu.cn

\title{
Abstract
}

Background. It has been proposed that numerosity perception is the cognitive underpinning of mathematics ability. However, the existence of the association between numerosity perception and mathematics ability is still under debate, especially in adults. The present study examined the relationship between numerosity perception and mathematics ability and the moderating role of dots number (i.e., the numerosity of items in dot set) in adults.

Methods. Sixty-four adult participants from Anshun University completed behavioral measures that tested numerosity perception of small numbers and large numbers, mathematics ability, inhibition ability, visual-spatial memory, and set-switching ability.

Results. We found that numerosity perception of small numbers correlated significantly with mathematical ability after controlling the influence of inhibition ability, visual-spatial memory, and set-switching ability, but numerosity perception of large numbers was not related to mathematics ability in adults.

Conclusions. These findings suggest that the dots number moderates the relationship between numerosity perception and mathematics ability in adults and may contribute to explaining the contradictory findings in the previous literature about the link between numerosity perception and mathematics ability.

\section{Introduction}

Mathematics ability is a unique cognitive ability for humans, which is essential to our everyday life. Yet, there is a subgroup of people who have trouble acquiring mathematics skills. What factors influence mathematics ability? Previous studies have found that many domain-general factors could predict individual differences in mathematics ability, including memory (Raghubar 
40 et al., 2010), executive function (Kroesbergen et al., 2009) and early language ability (Hecht et 41 al., 2001). In addition, symbolic number system (SNS) ability was an essential domain-specific 42 predictor of mathematical ability (Lau et al., 2021; Lyons et al., 2018). For example, Geary and 43 vanMarle (2016) found that young children's core symbolic abilities significantly correlated with later mathematics achievement. Recently, several studies have found a potential role of numerosity perception in facilitating individuals' formal mathematical skills (e.g., Elliott et al., 2019; Halberda et al., 2008; Mazzocco et al., 2011; Park \& Brannon, 2013). Furthermore, numerosity perception has been found to be a more important predictor of mathematics ability than other general cognitive functions (Chen \& Li, 2014; Halberda et al., 2008).

Numerosity perception is the elementary numerical ability in both humans and animals, representing nonverbal information of quantity without counting (Dehaene, 1997; Dehaene \& Changeux, 1993; Feigenson et al., 2004). A numerosity discrimination task is commonly applied to measure the precision of numerosity perception in which two dot sets are displayed briefly, and the participants are asked to judge which set is more numerous (Halberda \& Feigenson, 2008; Inglis et al., 2011). The numerosity discrimination performance, such as weber fraction (w), was used as an index of numerosity perception. The $\mathrm{w}$ is an abstract measure of discrimination threshold that specifies the precision of the numerosity representation and represents the width of numerosity tuning curve (Kersey \& Cantlon, 2017; Piazza et al., 2004), where higher $\mathrm{w}$ reflects less accurate numerosity representation.

The supporting evidence for the relationship between numerosity perception and mathematics ability came from the fact that numerosity discrimination performance (which reflects numerosity perception) correlated with mathematics ability (Halberda et al., 2008; Libertus et al., 2011; Mazzocco et al., 2011), which was measured by different tasks (Holloway \& Ansari, 2009; Fagerlin et al., 2007). Furthermore, it has been shown that training on numerosity perception tasks improved formal mathematics abilities in both children and adults (Hyde et al., 2014; Park $\&$ Brannon, 2013). However, it should be noted that many studies did not find such a relationship (Gilmore et al., 2013; Lyons \& Beilock, 2011; Lyons et al., 2014). The inconsistent results might ascribe to two reasons. First, the inconsistency might be caused by age differences of participants recruited (Gilmore et al., 2013; Inglis et al., 2011; Schneider et al., 2017). For instance, Inglis et al. (2011) found that the association between numerosity perception and mathematics skills only existed in children but not in adults. Second, a methodological difference which refers to the different numerosity of items in the dot set (i.e., dots number) used in previous studies, might also contribute to the inconsistency. There is evidence that the number of stimuli that has to be estimated (i.e., dots number) could influence the perception mechanism (Anobile et al., 2016; Anobile et al., 2014; Anobile et al., 2016; Pome et al., 2019; Fornaciai \& Park, 2017; Zimmermann, 2018). It seems that small numbers of stimuli (relatively sparse dotpattern) were sensed by the numerosity mechanism, but large numbers (relatively dense dotpattern) were sensed by the density-texture mechanism (Anobile et al., 2016; Anobile et al., 
80

81

82

83

84

85

86

87

88

89

90

91

92

93

94

95

96

97

98

99

100

101

102

103

104

105

106

107

108

109

110

111

112

113

114

115

116

117

118

119

2014; Zimmermann, 2018). Actually, previous studies found that only the numerosity perception of small numbers correlated with mathematics ability in children (Anobile et al., 2016).

Given the above, this study aimed to investigate the relationship between numerosity perception and mathematics ability and the effect of dots number on this relationship in adults. In particular, we conducted three measurements to assess participants' mathematics ability, including an arithmetical test, a subjective mathematics ability evaluation, and their mathematics course scores. In the meantime, we conducted the numerosity discrimination tasks of small and large number conditions separately, which allowed us to evaluate the numerosity perception of different dot numbers. In addition, to control the potential effects of other general cognitive abilities on the relationship between numerosity perception and mathematics ability (Fuhs \& McNeil, 2013; Gilmore et al., 2013), all participants performed three measurements on their inhibition ability, visual-spatial memory, and set-switching ability. We predicted that the number of dots would moderate the relationship between numerosity perception and mathematics ability in adults, and only the numerosity perception of small numbers would be related to mathematics ability.

\section{Materials \& Methods}

\section{Participants}

Sixty-four undergraduate students from Anshun University ( 28 males and 36 females, Mage $=$ 20.27, SDage = 1.11) participated in this study and received financial compensation for their time. This study was approved by the ethics committee of the School of Education Science, Anshun University (ID number: ASU-JYXY-201903) and all participants provided written informed consent at the beginning of the experiment. Three participants were excluded from the analyses due to either misunderstanding experimental task demand or missing data caused by computer errors.

\section{Apparatus and software}

All measurements were completed in a dimly lit room. Computer-based experiments were completed using MATLAB (R2016b, MathWorks, Cambridge, MA) and PsychToolbox (Brainard, 1997) on a 23-inch LED monitor (Dell: U2312 HM) with 1,600 × 1,080 resolution at $60 \mathrm{~Hz}$ and a standard desk (width: $70 \mathrm{~cm}$; length: $150 \mathrm{~cm}$ ), viewed binocularly from a distance of $60 \mathrm{~cm}$.

\section{Measures}

Numerosity perception, mathematics ability, inhibition ability, visual-spatial memory, and setswitching ability were measured in the following sequential order. The whole experimental procedure took approximately 85 minutes.

Numerosity perception. A modified version of a numerosity discrimination task adapted from Anobile et al. (2014) was used to measure numerosity perception. Two sets of dots were of equal 
120

121

122

123

124

125

126

127

128

129

130

131

132

133

134

135

136

137

138

139

140

141

142

143

144

145

146

147

148

149

150

151

152

153

154

155

156

157

158

159

size and presented simultaneously for $250 \mathrm{~ms}$ on either left or right side of a central fixation point. Each set was constrained to a $14^{\circ}$-diameter virtual circle and comprised a number of $0.3^{\circ}$ (in visual angle) diameter dots, half white and half black. For each trial, the set on the right side of the fixation point was the reference, and the left set was the probe. In separate blocks, the reference always contained 11 dots (small number condition) or 152 dots (large number condition), while the numerosity of the probe was changed from trial to trial, following the Method of Constant stimuli that varied the number of dots from $50 \%$ to $200 \%$ of the number of the reference that was split into seven equal log unit steps. For small number condition, the probe patches contained $6,7,9,11,14,17$, or 22 dots. For large number condition, the probe patches contained 76, 96, 121, 152, 192, 241, or 304 dots. Each of the seven probe stimulus levels was tested for 16 trials, and each block consisted of 112 trials. For each trial, participants were asked to judge as accurately and quickly as possible which set had more dots. Two blocks were presented in counterbalanced order. Participants gave their responses using a keyboard. Based on the pilot study, 14 practice trials were given to participants with feedback before the formal data collection.

"The proportion of probe greater trials" was plotted against the reference number and fitted with the Methods of Probits (Sun et al., 2013). The 50\% point estimated the point of subjective equality (PSE), and the difference in numerosity between the $25 \%$ and $75 \%$ points provided the just notable difference (JND), which was then divided by PSE to estimate the Weber fraction (w).

Mathematics ability. Three measures were used to assess participants' mathematics ability, including a modified-version arithmetical test (AT), subjective mathematics ability evaluation, and mathematics course score. The arithmetical test, which was designed based on Shalev et al. (2001), assessed basic arithmetic skills. The types of items that were tested included number facts, complex arithmetic, decimals calculation, and fraction calculation. In the self-developed measure of subjective mathematics ability evaluation, participants were instructed to evaluate their mathematics ability compared with other people using a 10 -point scale $(1=$ the worst level, $10=$ the best level). Furthermore, participants' college entrance examination score was used as the mathematics course score. Following the method of convention of Holloway and Ansari (2009), the scores for the three tasks were converted into z-scores and averaged to create an overall mathematics ability score for each participant, which was then used in the following analyses as an index of participants' mathematics ability.

Inhibition ability. Inhibition ability is the ability to suppress dominant, automatic, or prepotent response for irrelevant or no-longer-relevant information (Toll et al., 2011). The classical Go/No-go task was used to assess inhibition ability (Falkenstein et al., 1999). In this task, participants were told to respond to a yellow square (Go stimuli) and stop responding to a yellow circle (No-go stimuli). Participants were given five practice trials with feedback. The official 
160

161

162

163

164

165

166

167

168

169

170

171

172

173

174

175

176

177

178

179

180

181

182

183

184

185

186

187

188

189

190

191

192

193

194

195

196

197

198

199

task had 200 test trials without feedback, including 150 Go trials and 50 No-go trials. For each participant, the accuracy for No-go trials was recorded as the inhibition score.

Visual-spatial memory. Visual-spatial memory is a system for retaining location and object information (Wood, 2011). To assess participants' visual-spatial memory ability, a widely used memory span task for visual patterns was administered (Mejias et al., 2012). The task was developed by Wilson et al. (1987) and consisted of the presentation of boxes with some boxes being filled in. The participants were instructed to remember the pattern of the boxes, which was displayed for $2 \mathrm{~s}$. After a blank interval of $2 \mathrm{~s}$, the boxes would be presented again with one box missing. The participants had to recall which box was missing. The initial pattern involved filling in two boxes, and upon receiving two correct responses out of three attempts, the complexity of the pattern would increase. The final number of successfully recalled boxes was recorded as a memory span score.

Set-switching ability. Set-switching ability is the ability to direct actions and thoughts to selected goals; it has been thought of as a kind of executive control. In this study, a modified Trail Making Test was presented in the paper-and-pencil version to measure set-switching ability (Arbuthnott \& Frank, 2000). The test consisted of three trials: Part A-1, Part A-2, and Part B. Part A-1 included drawing a line connecting consecutive numbers from 1 to 25 . Part A-2 included drawing a line connecting consecutive letters from A to Y. Part B included drawing a line connecting alternating numbers and letters in sequence (i.e., 1-A-2-B and so on). Participants were shown three trials in order. In each trial, a timer was set when the participants began drawing a line on paper and stopped when they put the pencil on the desk. The time to complete each trial was recorded. The ratio of completion time between Part B and Part A (average completion time of Part A-1 and Part A-2) was calculated as a set-switching score. A higher set-switching score indicated a poorer set-switching ability.

\section{Data analysis}

The statistical analyses of data were performed using SPSS software (Version 23.0). The correlations between all variables were measured using Zero-order correlation analyses. Then, we used hierarchical regression analysis to explore the predictors of mathematics ability.

\section{Results}

\section{Mathematics ability score}

We looked for correlations of the participants' performance on the three mathematics ability tests. Subjective mathematics ability evaluation was significantly correlated with AT accuracy ( $r$ $=0.32, p=0.013)$ and the mathematics course score $(r=0.32, p=0.012)$. However, AT accuracy was not significantly correlated with mathematics course scores, $r=0.15, p=0.257$.

Given that mathematics ability is a complex construct and includes different skills (Mannamaa et al., 2012), these results showed that performances in three tests measured not only common 
200

201

202

203

204

205

206

207

208

209

210

211

212

213

214

215

216

217

218

219

220

221

222

223

224

225

226

227

228

229

230

231

232

233

234

235

236

237

238

239

240

aspects but also different aspects of mathematics ability. This might, in part, explain the previous contradictory relationships between numerosity judgments and mathematics ability. To provide an evaluation of overall mathematics ability, we followed Holloway and Ansari (2009) and used the mathematics ability score for each participant in further analyses.

\section{The correlations between numerosity perception and mathematics ability}

A zero-order correlation matrix was calculated to investigate the relationship between all measurements (See Table 1). Results showed that mathematics ability significantly correlated with $w_{\text {small }}(r=-0.42, p=0.001$; the alternative Pearson $r=-0.36, p=0.004$, see Figure 1), but not with $w_{\text {large }}(r=0.10, p=0.444$; the alternative Pearson $r=-0.11, p=0.414)$, indicating that lower mathematical ability was associated with higher $w$ (reflecting poorer numerosity discrimination precision) in the small number condition. Further correlation analysis showed that $w_{\text {small }}$ was significantly correlated with AT accuracy and the mathematics course score $(r=-0.32$, $p=0.017 ; r=-0.37, p=0.005)$, but not with subjective mathematics ability evaluation $(r=$ $0.21, p=0.125)$. Conversely, we found no significant correlations between $w_{\text {large }}$ and three mathematics ability tests, $r \mathrm{~s}<0.24, p \mathrm{~s}>0.075$. It should be noted that there was also a significant correlation between $w_{\text {small }}$ and $w_{\text {large }}(r=0.47, p<0.001$; the alternative Pearson $r=$ $0.53, p<0.001$ ). Here, we further examined the unique effect of dots number (i.e., small number and large number conditions) on the relationship between numerosity perception and mathematics ability. We used a specific test of the difference between two dependent correlations with one variable in common developed by Lee and Preacher (2013) to compare the correlations between $w_{\text {small }}, w_{\text {large }}$ and mathematics ability. Results showed that there was a significant difference between the correlation of $w_{\text {small }}$ and mathematics ability and the correlation of $w_{\text {large }}$ and mathematics ability, $p<0.001$. These results indicated the dots number moderated the relationship between numerosity perception and mathematics ability.

(Insert Table 1. Descriptive statistics and Zero-order correlation)

\section{The correlations among other variables}

The correlation between visual-spatial memory and mathematics ability reached marginal significance $(r=0.26, p=0.054)$. Inhibition ability significantly correlated with $w_{\text {small }}(r=-0.27$, $p=0.044)$ but not with $w_{\text {large }}(r=-0.15, p=0.273)$. The correlations between all other variables also did not reach significance $(|r s|<0.19$, ps $>0.149)$.

(Insert Figure 1. The Pearson correlations between mathematics ability score and $w \mathrm{~s}\left(w_{\text {small }}\right.$ and $\left.w_{\text {large }}\right)$. A filled symbol represents a participant's original data. See Table 1 for zero-order correlations.)

\section{Hierarchical regression analyses}

To measure the extent to which mathematics ability could be predicted by numerosity perception of small numbers, we ran a hierarchical regression analysis with $w_{\text {small }}$ as a predictor and 
241 mathematics ability as the dependent variable. The $w_{\text {large }}$, visual-spatial memory, inhibition

242 ability, and set-switching ability were entered as control variables. As shown in Table 2, all

243 controlling variables only explained $8 \%$ of mathematics ability variance, $F_{\text {change (56) }}=1.24, p=$

244 0.304. The numerosity perception of small numbers $\left(w_{\text {small }}\right)$ still explained a significant portion

245 of mathematics ability variance $(17 \%), F_{\text {change }(55)}=12.06, p=0.001$.

246

247 (Insert Table 2. Hierarchical regression analysis)

248

249 Discussion

250

251

252

253

254

255

256

257

258

259

260

261

262

263

264

265

266

267

268

269

270

271

272

273

274

275

276

277

278

279

280

The present study examined the relationship between numerosity perception and mathematics ability in adults and found the moderating role of dots number in this relationship. We showed that a significant association existed only between numerosity discrimination of small numbers and mathematics ability in adults. Even after controlling other cognitive variables, the association remained stable and robust.

Prior studies have not found a significant correlation between numerosity perception and mathematics ability in adults (Gilmore et al., 2013; Inglis et al., 2011). However, the inconsistency might be attributed to that many of these studies did not take into account the specific effect of methodological differences in numerosity perception tasks and other cognitive abilities (Dietrich et al., 2019; Fuhs \& McNeil, 2013; Norris \& Castronovo, 2016). The present study found that the relationship between numerosity perception and mathematics ability might depend on the dots number. In addition, even after controlling other cognitive variables (i.e., inhibition ability, visual-spatial memory, and set-switching ability), the numerosity perception of small numbers could still explain $17 \%$ of mathematics ability variance. These results were consistent with a similar study in children (Anobile et al., 2016), in which only small numerosity perception thresholds were significantly correlated with mathematics ability. Indeed, it is suggested that small numerosity perception was based on numerosity mechanism, and large numerosity perception was based on density mechanism (Anobile et al., 2014; Zimmermann, 2018). Thus, our findings supported the notion that dots number could influence the association between numerosity perception and mathematics ability in adults. Future studies with more levels of number of dots are required in order to further explore how dots number influences the relationship between numerosity perception and mathematics ability. In addition, our current findings may have particular practical implications for math learning. They can guide psychological interventions to help children and adults with math learning difficulties improve mathematics ability.

In this study, the role of cognitive abilities in the relationship between numerosity perception and mathematics ability, particularly the inhibition ability, has not been validated. Fuhs and McNeil (2013) found correlations between numerosity perception, inhibition ability, and mathematics ability. They argued that the numerosity perception explained the mathematical achievement

Peer) reviewing PDF | (2021:09:65847:1:1:NEW 8 Nov 2021) 
281

282

283

284

285

286

287

288

289

290

291

292

293

294

295

296

297

298

299

300

301

302

303

304

305

306

307

308

309

310

311

312

313

314

315

316

317

318

319

320

only when considering the contribution of inhibition ability. Our study only found correlations between numerosity perception of small numbers and mathematics ability but not between inhibition ability and mathematics skills. One possibility was that the participants' age influenced the relationship between the two constructs. Several studies supported the relationship between inhibition ability and mathematics ability in preschool and school-age children (Bull \& Scerif, 2001). Yet, the nature of inhibition ability and mathematics ability profoundly changed with age (Espy et al., 2004). These variations inherently affected how inhibition ability related to mathematics ability (Ahmed et al., 2019). As discussed above, we speculated that the association of numerosity perception and mathematics ability might be stable and robust and exist in both children and adults; whereas the association of inhibition ability and mathematics ability might be diverse and labile and change with age. Future research could examine this issue.

Another critical finding pertained the selective relationship between inhibition ability and numerosity perception of small numbers. The results implied that adults with high levels of inhibition ability could better cope with small numerosity perception tasks. Adults needed to exact abstract numerical properties and inhibit extraneous continuous properties in small number tasks. But they only needed to exact continuous properties to complete large number tasks (Fuhs \& McNeil, 2013). It was not surprising that inhibition ability played a key role in numerosity perception of small numbers. In addition, although the correlation between visual-spatial memory and mathematics ability was significant in children (Caviola et al., 2020), the present study found a marginal association in adults. We speculated that the relationship between visualspatial memory and mathematics ability would be much less pronounced in adults since they had many years of schooling.

It was also important to note that the numerosity perception performances across both conditions were similar in the present study. These findings contradicted with the results of Anobile et al. (2014), who found that the numerosity perception threshold of small numbers was lower compared to large numbers. These inconsistencies could be partially attributed to individual differences in numerosity perception performance (Halberda et al., 2008; Ross, 2003). For example, Ross (2003) used numbers from 8 to 64 as base numbers and asked participants to complete a numerosity discrimination task. Four of five observers' thresholds initially increased almost linearly with base numbers and decreased with base numbers of 30 or above. However, the thresholds of one observer were consistently higher than any of the others and increased with base numbers. The author suggested that there might not be a universally available numerosity discrimination pattern. Given the large individual differences in patterns of numerosity perception, the differences in numerosity perception performance between the small and large number conditions might be cancelled out in the present study. Despite the lack of significant differences between small and large numerosity perception, only small numerosity perception correlated with mathematics ability and inhibition ability. These results implied that there might be separate mechanisms for small and large numbers, but more sensitive indexes other than the 
321

322

323

324

325

326

327

328

329

330

331

332

333

334

335

336

337

338

339

340

341

342

343

344

345

346

347

348

349

350

351

352

353

354

355

356

357

358

359

360

361

numerosity perception threshold were needed to detect the difference between small and large number perception. In addition, it appeared likely that the difference between psychophysical methods used by the previous study and the current study to measure $w$ could explain the inconsistent findings. Specifically, Anobile et al. (2014) used the adaptive Quest method, and we used the Method of Constant Stimuli. Future studies should compare the two different methods.

\section{Conclusions}

This article demonstrated the relationship between numerosity perception and mathematics ability in adults and the influence of dots number on this relationship. The results suggested that mathematics ability was significantly associated with the numerosity perception of small numbers rather than large numbers. It seemed likely that the numerosity perception of small and large numbers were encoded differently and had a distinct effect on mathematics ability. Future studies should consider other potential domain-specific factors, such as SNS, to further explore the relationship between numerosity perception and mathematics ability. In addition, the neural mechanisms underlying small and large numerosity perception might also provide strong evidence for the effect of dots number and should be explored further.

\section{Acknowledgements}

We would like to thank our participants who devoted their time to support this research and Feng Kong, Annebella Tsz Ho Choi, Zumei Wu, Yuezhen Li, Yingchun Wu, Qinglin Zhao for their assistance in data collection and manuscript preparation.

\section{References}

Ahmed, S. F., Tang, S., Waters, N. E., \& Davis-Kean, P. (2019). Executive Function and Academic Achievement: Longitudinal Relations From Early Childhood to Adolescence. Journal of Educational Psychology, 111(3), 446-458. doi: 10.1037/edu0000296

Anobile, G., Castaldi, E., Turi, M., Tinelli, F., \& Burr, D. C. (2016). Numerosity but not Texture-Density Discrimination Correlates With Math Ability in Children. Developmental Psychology, 52(8), 1206-1216. doi: $10.1037 /$ dev0000155

Anobile, G., Cicchini, G. M., \& Burr, D. C. (2014). Separate Mechanisms for Perception of Numerosity and Density. Psychological Science, 25(1), 265-270. doi: 10.1177/0956797613501520

Anobile, G., Cicchini, G. M., \& Burr, D. C. (2016). Number As a Primary Perceptual Attribute: A Review. Perception, 45(1-2SI), 5-31. doi: 10.1177/0301006615602599

Arbuthnott, K., \& Frank, J. (2000). Trail making test, part B as a measure of executive control: Validation using a set-switching paradigm. Journal of Clinical and Experimental Neuropsychology, 22(4), 518-528. doi: 10.1076/1380-3395(200008)22:4;1-0;FT518

Brainard, D. H. (1997). The psychophysics toolbox. Spatial Vision, 10(4), 433-436.

Bull, R., \& Scerif, G. (2001). Executive functioning as a predictor of children's mathematics ability: Inhibition, switching, and working memory. Developmental Neuropsychology, 19(3), 273-293. doi: 10.1207/S15326942DN1903_3 
362

363

364

365

366

367

368

369

370

371

372

373

374

375

376

377

378

379

380

381

382

383

384

385

386

387

388

389

390

391

392

393

394

395

396

397

398

399

400

401

402

403

404

405

406

407

408

409

410

Chen, Q., \& Li, J. (2014). Association between individual differences in non-symbolic number acuity and math performance: A meta-analysis. Acta Psychologica, 148, 163-172. doi: 10.1016/j.actpsy.2014.01.016

Caviola, S., Colling, L. J., Mammarella, I. C., \& Dénes Szcs. (2020). Predictors of mathematics in primary school: magnitude comparison, verbal and spatial working memory measures. Developmental Science, 23(6), 1-19. doi: 10.1111/desc. 12957

Dehaene, S. (1997). The number sense: how the mind creates mathematics. New York: Oxford University Press.

Dehaene, S., \& Changeux, J. P. (1993). Development of elementary numerical abilities - a neuronal model. Journal of Cognitive Neuroscience, 5(4), 390-407. doi: 10.1162/jocn.1993.5.4.390

Dietrich, J. F., Nuerk, H., Klein, E., Moeller, K., \& Huber, S. (2019). Set size influences the relationship between ANS acuity and math performance: a result of different strategies? Psychological Research, 83(3), 590612. doi: 10.1007/s00426-017-0907-1

Elliott, L., Feigenson, L., Halberda, J., \& Libertus, M. E. (2019). Bidirectional, Longitudinal Associations Between Math Ability and Approximate Number System Precision in Childhood. Journal of Cognition and Development, 20(1), 56-74. doi: 10.1080/15248372.2018.1551218

Espy, K. A., McDiarmid, M. M., Cwik, M. F., Stalets, M. M., Hamby, A.,... Senn, T. E. (2004). The contribution of executive functions to emergent mathematic skills in preschool children. Developmental Neuropsychology, 26(1), 465-486. doi: 10.1207/s15326942dn2601_6

Fagerlin, A., Zikmund-Fisher, B. J., Ubel, P. A., Jankovic, A., Derry, H. A.,... Smith, D. M. (2007). Measuring numeracy without a Math test: Development of the subjective numeracy scale. Medical Decision Making, 27(5), 672-680. doi: 10.1177/0272989x07304449

Falkenstein, M., Hoormann, J., \& Hohnsbein, J. (1999). ERP components in Go Nogo tasks and their relation to inhibition. Acta Psychologica, 101(2-3), 267-291. doi: 10.1016/S0001-6918(99)00008-6

Feigenson, L., Dehaene, S., \& Spelke, E. (2004). Core systems of number. Trends in Cognitive Sciences, 8(7), 307-314. doi: 10.1016/j.tics.2004.05.002

Fornaciai, M., \& Park, J. (2017). Distinct Neural Signatures for Very Small and Very Large Numerosities. Frontiers in Human Neuroscience, 11. doi: 10.3389/fnhum.2017.00021

Fuhs, M. W., \& McNeil, N. M. (2013). ANS acuity and mathematics ability in preschoolers from low-income homes: contributions of inhibitory control. Developmental Science, 16(1), 136-148. doi: 10.1111/desc.12013

Geary, D. C., \& Vanmarle, K. (2016). Young children's core symbolic and nonsymbolic quantitative knowledge in the prediction of later mathematics achievement. Developmental Psychology, 52(12), 2130-2144. doi: 10.1037/dev0000214

Gilmore, C., Attridge, N., Clayton, S., Cragg, L., Johnson, S., Marlow, N.,... Inglis, M. (2013). Individual Differences in Inhibitory Control, Not Non-Verbal Number Acuity, Correlate with Mathematics Achievement. Plos One, 8(6): e67374. doi: 10.1371/journal.pone.0067374

Halberda, J., Mazzocco, M. M. M., \& Feigenson, L. (2008). Individual differences in non-verbal number acuity correlate with maths achievement. Nature, 455(7213), 662-665. doi: 10.1038/nature07246

Halberda, J., \& Feigenson, L. (2008). Developmental change in the acuity of the "Number sense": The approximate number system in 3-, 4-, 5-, and 6-year-olds and adults. Developmental Psychology, 44(5), 1457-1465. doi: $10.1037 / \mathrm{a} 0012682$

Hecht, S. A., Torgesen, J. K., Wagner, R. K., \& Rashotte, C. A. (2001). The relations between phonological processing abilities and emerging individual differences in mathematical computation skills: A longitudinal study from second to fifth grades. Journal of Experimental Child Psychology, 79(2), 192-227. doi: 10.1006/jecp.2000.2586

Holloway, I. D., \& Ansari, D. (2009). Mapping numerical magnitudes onto symbols: The numerical distance effect and individual differences in children's mathematics achievement. Journal of Experimental Child Psychology, 103(1), 1729. doi: 10.1016/j.jecp.2008.04.001

Hyde, D. C., Khanum, S., \& Spelke, E. S. (2014). Brief non-symbolic, approximate number practice enhances subsequent exact symbolic arithmetic in children. Cognition, 131(1), 92-107. doi: 10.1016/j.cognition.2013.12.007

Inglis, M., Attridge, N., Batchelor, S., \& Gilmore, C. (2011). Non-verbal number acuity correlates with symbolic mathematics achievement: But only in children. Psychonomic Bulletin \& Review, 18(6), 1222-1229. doi: 10.3758/s13423011-0154-1 
411

412

413

414

415

416

417

418

419

420

421

422

423

424

425

426

427

428

429

430

431

432

433

434

435

436

437

438

439

440

441

442

443

444

445

446

447

448

449

450

451

452

453

454

455

456

457

458

459

Kersey, A. J., \& Cantlon, J. F. (2017). Neural Tuning to Numerosity Relates to Perceptual Tuning in 3-6-YearOld Children. The Journal of Neuroscience, 37(3), 512-522. doi: 10.1523/JNEUROSCI.0065-16.2017

Kingdom, F. A. A., \& Prins, N. (2010). Psychophysics: A practical introduction. San Diego: Elsevier Academic Press.

Klein, S. A. (2001). Measuring, estimating, and understanding the psychometric function: A commentary. Perception \& Psychophysics, 63(8), 1421-1455. doi: 10.3758/BF03194552

Kroesbergen, E. H., Van Luit, J. E. H., Van Lieshout, E. C. D. M., Van Loosbroek, E., \& Van de Rijt, B. A. M. (2009). Individual Differences in Early Numeracy The Role of Executive Functions and Subitizing. Journal Of Psychoeducational Assessment, 27(3SI), 226-236. doi: 10.1177/0734282908330586

Lau, N. T. T.; Merkley, R., Tremblay, P., Zhang, S., Jesus, S. D., \& Ansari, D. (2021). Kindergarteners' symbolic number abilities predict nonsymbolic number abilities and math achievement in grade 1. Developmental Psychology, 57(4), 471-488. doi: 10.1037/dev0001158

Lee, I. A., \& Preacher, K. J. (2013, September). Calculation for the test of the difference between two dependent correlations with one variable in common [Computer software]. Available from http://quantpsy.org.

Libertus, M. E., Feigenson, L., \& Halberda, J. (2011). Preschool acuity of the approximate number system correlates with school math ability. Developmental Science, 14(6), 1292-1300. doi: 10.1111/j.1467-

7687.2011.01080.x

Lyons, I. M., Price, G. R., Vaessen, A., Blomert, L., \& Ansari, D. (2014). Numerical predictors of arithmetic success in grades 1-6. Developmental Science, 17(5), 714-726. doi: 10.1111/desc.12152

Lyons, I. M., \& Beilock, S. L. (2011). Numerical ordering ability mediates the relation between number-sense and arithmetic competence. Cognition, 121(2), 256-261. doi: 10.1016/j.cognition.2011.07.009

Lyons, I. M., Bugden, S., Zheng, S., Jesus, S. D., \& Ansari, D. (2018). Symbolic number skills predict growth in nonsymbolic number skills in kindergarteners. Developmental Psychology, 54(3), 440-457. doi: 10.1037/dev0000445

Mannamaa, M., Kikasb, E., Peets, K., \& Palu, A. (2012). Cognitive correlates of math skills in third-grade students. Educational Psychology, 32(1), 21-44. doi: 10.1080/01443410.2011.621713

Mazzocco, M. M. M., Feigenson, L., \& Halberda, J. (2011). Preschoolers' Precision of the Approximate Number System Predicts Later School Mathematics Performance. Plos One, 6(9). doi: 10.1371/journal.pone.0023749

Mejias, S., Gregoire, J., \& Noel, M. (2012). Numerical estimation in adults with and without developmental dyscalculia. Learning and Individual Differences, 22(1), 164-170. doi: 10.1016/j.lindif.2011.09.013

Norris, J. E., \& Castronovo, J. (2016). Dot Display Affects Approximate Number System Acuity and Relationships with Mathematical Achievement and Inhibitory Control. Plos One, 11(5). doi: 10.1371/journal.pone. 0155543

Park, J., \& Brannon, E. M. (2013). Training the Approximate Number System Improves Math Proficiency. Psychological Science, 24(10), 2013-2019. doi: 10.1177/0956797613482944

Piazza, M., Izard, V., Pinel, P., Le Bihan, D., \& Dehaene, S. (2004). Tuning curves for approximate numerosity in the human intraparietal sulcus. Neuron, 44(3), 547-555. doi: 10.1016/j.neuron.2004.10.014

Pome, A., Anobile, G., Cicchini, G. M., \& Burr, D. C. (2019). Different reaction-times for subitizing, estimation, and texture. Journal of Vision, 19(6), 1-9. doi: 10.1167/19.6.14

Raghubar, K. P., Barnes, M. A., \& Hecht, S. A. (2010). Working memory and mathematics: A review of developmental, individual difference, and cognitive approaches. Learning and Individual Differences, 20(2SI), 110122. doi: 10.1016/j.lindif.2009.10.005

Ross, J. (2003). Visual discrimination of number without counting. Perception, 32(7), 867-870. doi: $10.1068 / \mathrm{p} 5029$

Schneider, M., Beeres, K., Coban, L., Merz, S., Schmidt, S. S., Stricker, J.,... De Smedt, B. (2017). Associations of non-symbolic and symbolic numerical magnitude processing with mathematical competence: a meta-analysis. Developmental Science, 20(3). doi: 10.1111/desc.12372

Shalev, R. S., Manor, O., Kerem, B., Ayali, M., Badichi, N., Friedlander, Y.,... Gross-Tsur, V. (2001). Developmental dyscalculia is a familial learning disability. Journal of Learning Disabilities, 34(1), 59-65. doi:

$10.1177 / 002221940103400105$

Peer] reviewing PDF | (2021:09:65847:1:1:NEW 8 Nov 2021) 
460

461

462

463

464

465

466

467

468

469

470

471

472

473

474
Sun, P., Gardner, J. L., Mauro, C., Kenichi, U., R Allen, W., Keiji, T.,... Kang, C. (2013). Demonstration of tuning to stimulus orientation in the human visual cortex: a high-resolution FMRI study with a novel continuous and periodic stimulation paradigm. Cerebral Cortex, 23(7), 1618-1629

Toll, S. W. M. , Van der Ven, S. H. G. , Kroesbergen, E. H. , \& Van Luit, J. E. H. . (2015). Executive functions as predictors of math learning disabilities. Journal of Learning Disabilities, 44(6), 521-532. doi: 10.1177/0022219410387302

Watson, A. B., \& Fitzhugh, A. (1990). The method of constant stimuli is inefficient. Perception \& Psychophysics, 47(1), 87-91. doi: 10.3758/BF03208169

Wilson, J. T. L., Scott, J. H., \& Power, K. G. (1987). Developmental differences in the span of visual memory for pattern. British Journal of Developmental Psychology, 5(3), 249-255

Wood, J. N. (2011). When do spatial and visual working memory interact?. Attention, Perception \& Psychophysics, 73, 420-439. doi: 10.3758/s13414-010-0048-8

Zimmermann, E. (2018). Small numbers are sensed directly, high numbers constructed from size and density. Cognition, 173, 1-7. doi: 10.1016/j.cognition.2017.12.003 
Figure 1

The Pearson correlations between mathematics ability score and $w s\left(w_{\text {small }}\right.$ and $\left.w_{\text {large }}\right)$.

A filled symbol represents a participant's original data. See Table 1 for zero-order correlations. $* * p<0.01$

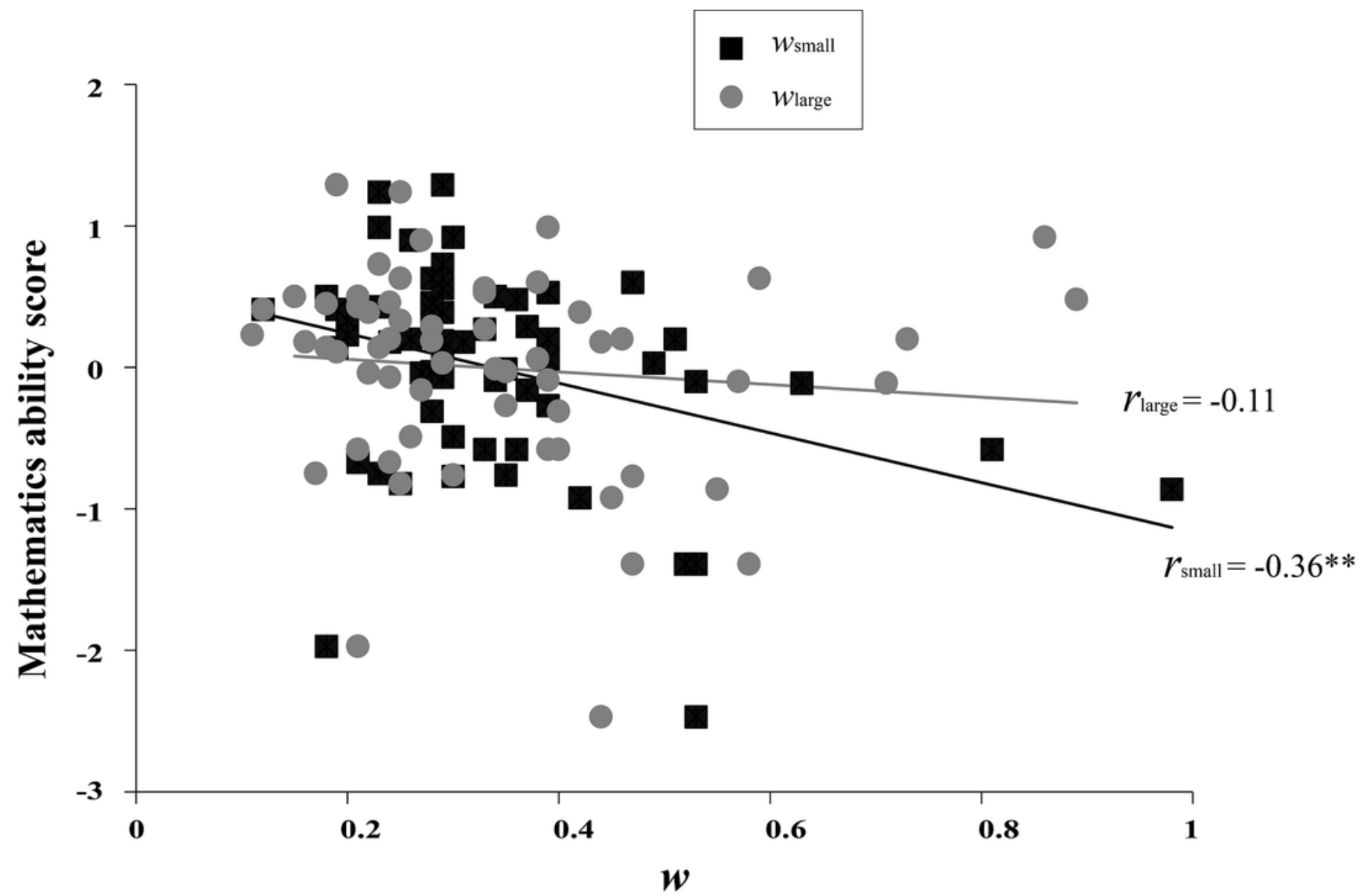


Table $\mathbf{1}$ (on next page)

Descriptive statistics and Zero-order correlation 
1 Table 1. Descriptive statistics and Zero-order correlation

\begin{tabular}{|c|c|c|c|c|c|c|c|c|c|}
\hline$\square$ & Range & $M$ & $S D$ & 1 & 2 & 3 & 4 & 5 & 6 \\
\hline 1. $w_{\text {small }}$ & $0.12-0.98$ & 0.34 & 0.15 & 1.00 & & & & & \\
\hline 2. $w_{\text {large }}$ & $0.11-0.89$ & 0.34 & 0.17 & $0.47^{* * *}$ & 1.00 & $\square$ & $\square$ & $\square$ & $\square$ \\
\hline $\begin{array}{l}\text { 3. Mathematics } \\
\text { ability }\end{array}$ & $-2.47-1.29$ & 0.00 & 0.71 & $-0.42^{* * *}$ & 0.10 & 1.00 & $\square$ & $\square$ & $\square$ \\
\hline $\begin{array}{l}\text { 4. Visual-spatial } \\
\text { memory }\end{array}$ & $2-16$ & 8.43 & 3.34 & 0.14 & -0.05 & 0.26 & 1.00 & $\square$ & $\square$ \\
\hline $\begin{array}{l}\text { 5. Inhibition } \\
\text { ability }\end{array}$ & $0.64-0.96$ & 0.84 & 0.08 & $-0.27^{*}$ & -0.15 & -0.19 & -0.03 & 1.00 & $\square$ \\
\hline $\begin{array}{l}\text { 6. Set-switching } \\
\text { ability }\end{array}$ & $0.07-5.35$ & 1.75 & 0.94 & 0.08 & -0.15 & 0.11 & 0.06 & 0.00 & 1.00 \\
\hline
\end{tabular}

2 Note. ${ }^{* * *} p<0.001 * * p<0.01 * p<0.05$ 
Table 2 (on next page)

Hierarchical regression analysis 
1 Table 2. Hierarchical regression analysis

\begin{tabular}{lcccccc}
\hline Model & Predictor & $R^{2}$ & $R_{\text {chang }}^{2}$ & $F_{\text {change }}$ & $d f$ & $P$ \\
\hline \multirow{2}{*}{ First step } & $\begin{array}{l}\text { visual-spatial memory, } \\
\text { Inhibition ability, Set- } \\
\text { Switching ability, } w_{\text {large }}\end{array}$ & 0.08 & - & 1.24 & 56 & 0.304 \\
Second step & $w_{\text {small }}$ & 0.25 & 0.17 & 12.06 & 55 & 0.001 \\
\hline
\end{tabular}

2 